\title{
Study on Style of Chinese Classical Dance and Personalized Creation
}

\author{
Xiaoya Long
}

Jishou University, Jishou Hunan, 416000, China

\begin{abstract}
Key words: Creation, Style, Classical dance, Personalized, Chinese.
\end{abstract}
\begin{abstract}
Chinese classical dance integrates the aesthetic characteristics of fresco, traditional Chinese opera, calligraphy, martial arts, etc., which is of a variety of artistic conceptions, ethos and images. Under the cultivation of various different arts and the drive of the history, the approaches, postures and movements of Chinese classical dance inherit the characters of classical culture, such as balance, elegance, preciseness, harmony, and standard, of special styling and strong rhythmical feeling, preserving the Chinese style, and becoming classics forever. "Elegance" and "harmony" are the two most important elements in dance form and aesthetic emotion. Dance movements are made to mainly show the meaning with body, exercising a combination of inflexibility and yielding and of appearance and spirit. Physical performances are soft, elegant, and coquettish. The cultural deposits are deep and the style and charm are elegant. Creation can be made without too much hesitation. The all-inclusive materials collected from various fields reflect the profound and insightful dance culture. This paper is intended to research the style and personalized creation of Chinese classical dance, with a purpose to inherit and develop Chinese classical dance.
\end{abstract}

\section{Introduction}

Chinese classical dance has experienced generations of systemization, processing, refinery, and creation, conflicts with, coordinates with, and blends with multiple cultural vogues, sentiments, and styles, has ancient and historical cultural traits, cultural spirits, artistic achievements, aesthetic pursuits and national ethos, and is featured by wide spreading, representativeness and classical. It mainly can be classified into dance in Chinese opera, religious dance, and court dance. It is one of the main dance types staged in China. Chinese classical dance covers the brilliant history of Chinese nation, has been handed down, evolved and spread for a long time, can convert vulgar into elegance, is mainly presented to sense organs, is to build idealized and super utilitarian-based body language, has a strong artistic appeal and cohesive force as a super utilitarian-based dance, and can sublime and purify audiences' spirit[1]. Chinese classical dance is mainly constituted by earthward, centripetal and inward movements that are poetic, exercise the combination of appearance and spirit, are developed on the basis of superior culture elements, are of unique lingering charm, cultural taste, aesthetic orientation, distinct style, last forever, and are of model significance. There are a lot of design methods of Chinese classical dance.

\section{Style}

\section{Body Figure Style}

Body figure is the main part showing the style of Chinese classical dance, which highly integrates four elements, i.e. rhythm, power, spirit and appearance. In body figure of dance, spirit accounts for $80 \%$, power for $60 \%$ and appearance for $30 \%$. The shape of body figure is mainly reflected via instantaneous state change, multiple movement rhythms, and three circular movements, showing the beauty of rhythm and figure. Circling is a typical component element of the shape of body figure, such as double shaking hands, hot wheels, turning between the waist. Turning between the waist refers to dedoubling around the waist as the axle, of which the movements mainly include "facing upward”, "throwing out”, "nodding”, "leaning”, and "rushing”. The lingering charm of double shaking hands is harmonious and rounded. The mode of dedoubling via hot wheels consists of 
vertical circular, obverse V-shaped circular, flat circular, and so on. The mode of dedoubling via walking is walking outside, starting from the waist and manipulated by the heart. The flowing process is not even, not straight, nor symmetrical. The spirit of body figure means "harmony between atmosphere and conception and between conception and idea", the power of body figure means "proper force, reserved, soft, vigorous, cadence, forceful, and at proper speed", the rhythm of body figure means "as vigorous and nimble as a flying dragon, unfolding must be followed by folding, rushing must be followed by leaning, complicated and confusing, drifting clouds and flowing waters, dynamic, and peaks rising one after another"[2]. The artistic styles of body figure include elegance, gentleness, rigorousness, and harmony. The Chinese classical dance is to present classical art via elegant body figure of dancers, who have elegant posture, and walk gently. Meanwhile, harmony and unity between time and space, and between reason and emotion are achieved, the language of dance is refreshing, elegant and gentle. Take the example of the "lotus dance". Performers do gentle dance steps in a smooth way, sometimes winding and sometimes circling round. Ripples rise, and flow, showing the beautiful, holy, pure, slim and graceful image of lotus. The dance scene is peaceful and tranquil, and is unforgettable. The form, content, feelings and ideas of body figure all should comply with classical aesthetic requirements, and depend on performers' professions, music, drumming, uniform and props. Every movement should follow certain rule, to show the beauty of harmony and classics. Thus, body figure art has the feature of rigorousness. Besides, body postures usually are both rigid and soft, static and dynamic, virtual and real, and positive and negative. The opposites interwork to create the beauty of balance and harmony[3]. Take the example of the Drunken Drum. The movements of spinor tiger jump, turning over at spot and black dragon twisting stake are circular, pitching, free, and twisting. The three-dimensional shape are both rigid and soft, and both feminine and manly, the rhythmical feeling is strong and it is smooth, and a realm of harmony and beauty is achieved.

\section{Artistic Conception Style}

Chinese classical dance integrates various artistic forms, including painting, calligraphy, poem, opera and martial art, and artistic conception is the source of life and an important representation of dance art. Spirit refers to air, anima and vigor, and rhyme refers to charm and lingering charm. Spirit shows that all things on earth are manly, while rhyme shows that all things on earth are gentle. For example, the spirit in the "moving hands like clouds" refers to crossing hands to show the beauty of manly dance, and rhyme refers to dedoubling to show the beauty of gentle dance; the pose making and wrist raising show charm and power incisively and vividly, and flowers in bud and inward convergence are perfectly unified[4]. The best states of artistic conception include meaningful lingering charm, elegant charm, refreshing, overflowing with vigor, and nadi tsa conversion. The artistic conception and style of Chinese classical dance is mainly to seek to be idyllic, empty, graceful, physical and metal concentrated, combination of spirit and appearance, and integration of interior and exterior. Specifically, the artistic conception style refers to being idyllic, conduct style refers to being empty and graceful, and the mood style refers to being physical and mental concentrated and upholds neutralization. Artistic conception is a dance spirit, and reflects philosophical concept. Chinese classical dance needs artistic conception as its core. Only by deeply exploring artistic conception can the cultural connotation of Chinese classical dance be enriched. The artistic conception of Chinese classical dance emerged in the Han dynasty. The beauty of artistic conception is reflected via harmony, apathy, and elegance. The aesthetic style and aesthetic form of artistic conception were gradually formed in the Wei and Jin dynasties, and reached the extreme in the Tang dynasty. The Chinese classical dance since the Song dynasty mainly focused on rhyme, and during the 100-year period since then, the artistic conception of Chinese classical dance faded out until the establishment of People's Republic of China. Since then, artistic conception was regard as the main perform method of Chinese classical dance art. For The Dance with Fan, body movements are full and smooth, body postures are like floating clouds and flowing water, the artistic conception is touching, dancers make movements full of changes at a proper speed, forming a contrast with the speed, gravity and range of 
folding fan. The artistic conception of calligraphy is perfectly showed, and the life rhythm of continuous reproduction breed in an endless succession and natural harmony is conveyed. The uniforms for dancers of the Wind Sing are mainly in white. Dancers' body movements are rigorous and smooth. Instantaneous motionless is always followed by dynamic movements. Dancers rotate nimbly and deftly, and jump gently and airily, showing the emptiness, grace, loneliness and drifting of artistic conception of the dance. To perform the Maid with Fan, the dancer wears a peaceful and elegant facial expression, and makes full and reserved body movements and gentle and elegant dance steps. This work conveys the emotional appeal and charm of traditional women. The artistic conception is idyllic.

\section{Romantic Charm Style}

Romantic charm refers to the unity of style, charm, expression and grace. Charm refers to grace, harmony and taste, while spirit refers to an ethos and spirit. Charm shows the gentle beauty, while spirit shows the manly beauty. Charm is achieved as long as spirit is showed, and charm and spirit promote each other. The romantic charm of Chinese classical dance has the features of dispersibility, abstraction and perceptibility. It seems to be straight, but is curved in fact. It is full and rotating, and gentle and graceful. Romantic charm runs throughout the process of dance performance. In the process of dance performance, romantic charm has not fixed form, is abstract, but leads aesthetic appreciation and has strong art aesthetic feeling and impact. Audiences can fully understand and perceive the romantic charm of dance. If Chinese classical dance loses its romantic charm, it also will lose its core soul. Perceptibility means that dancers can convey the artistic theme via a series of dance movements, to transform abstract romantic charm into perceptible things. In Chinese classical dance, romantic charm and body figure are closely linked to convey the soul of dance via visual beauty[5]. Take the example of the Guerrillas Dance. The formation of the dancing team is in triangle, dancers move forward or backward and left and right to create a violent war scene in reed marshes, to convey the romantic charm of hard fighting of guerrillas. In the Vintage Legacy, the main dance pose is Thousand-Hand Kwan-yin. Its artistic conception is complicated and confusing, and time-honored. This work has brought audiences into the mysterious, magnificent, solemn and primitive ancient world of Dunhuang Grottos by creating artistic conception and romantic charm. For example again, in the Zhaojun Going Abroad, the dancers turned over and rotated repeatedly, to convey the emotional and psychological changes of Wang Zhaojun. Audiences can feel Zhaojun's loneliness, helplessness, grievousness, and uneasiness on the way. Besides, the romantic charm of Chinese classical dance also emphasizes Yin and Yang. For example, the Pangu Dance blends the styles of boldness, unconstrainedness, and grace and elegance, in which the spirit and charm promote each other, and flexibility and inflexibility are combined. The Dance of the White Snake is roundabout, lingering, elegant, and misty, touching audiences, and conveying vivid and lively romantic charm.

\section{Personalized Creation}

\section{Theme}

The creation themes have the direct bearing on cultural taste. For theme selection, national personalities should go before creative personalities, both cultural taste and artistic appeal should be considered, the relationship between elegance and vulgar should be handled well, it is not allowed to purely seek sensory stimuli or flattering, and instead themes that can reflect historical sense and historic morphology should be selected. The artistic temperament, cultural form, language and image should be faithful to the history as far as possible, so as to ensure the aesthetic meaning and cultural emotional appeal of the themes[6]. The Tung Chueh Chi is themed by the miserable life of dancers of the Bronze Sparrow Terrace, revealing the tragic fate of dancers, and showing the inhumanity and ruthlessness of the feudal rule society represented by bigwigs. The Tung Chueh Chi is based on the history, but not limited to the history. It is full of Chinese charm and style, and fully reflects national spirit and cultural deposits. On the other hand, the themes of personalized creation of Chinese 
classical dance are gradually approaching the public aesthetic requirements, having broken the routine of young men and women who are deeply in love but unable to fulfill their passion or the lasting affection of boys and girls. Instead, the creation resources are constantly developed and the artistic vision has been constantly broadened, so that the themes are closer to our life and the time, reflect the spirit of the times, are of national character and sociality, and become popular to the public. The public aesthetic needs, such as dance costume beauty, and dancer figure and looks, will have an impact on creation themes. In the event that aesthetic needs change, besides the spiritual meaning and cultural meaning, time significance also should be taken into account at the time of theme selection. For example, the Terracotta Warrior Soul was adapted from the Emperor Qin Calling up Troops. The history of Terracotta Figures of Qin Dynasty is well known to the public, so the content of the Terracotta Warrior Soul can arouse sympathy. The producer director is imaginative and has distinct creative train of thought, presenting a new artistic image of Terracotta Figures of Qin Dynasty. For example again, the Kong Yiji, based on a novel popular to the public, breaks the pathetic, plaintive and tedious situation of Chinese classical dance. The dance form is funny, humorous and harmonious, and body language is made use of to revive the literary figures. The dance consists of three parts, i.e. book stealing, drinking and leg breaking. The choreographer makes use of development element, enhancement element and extraction element to give vitality and expressive force to figures. Bouncing and jumping occur repeatedly, and the dance vocabularies are humorous[7].

\section{Artistic Conception}

Dance is a cultural form, and the breath, rhythm and styling should reflect cultural connotation and implication. Thus, the creation of Chinese classical dance includes design of flowing dancing postures, body postures and movements, and arrangement of dance form and image, to make choreographic work have artistic conception, render the feelings, emotional appeal, interest and charm and mood via contrast between true and false, between virtual and real and between static and dynamic, and enhance the spiritual, ideological, logical and formal beauty of dance work. To create the artistic conception of Chinese classical dance, it is recommended to design characters with distinct personalities. For example, for the Lane in A Rainy Town, the character is a girl with an oiled paper umbrella in a rainy town in south China. The girl is as pretty as peach blossom, wears cheongsam, and walks enchantingly, which is liable to make audiences to imagine tender and elegant girls in the regions south of the Yangtze River. The dance is set in an ancient town in south China far from the madding crowd, rainwater drops on the green flagstone road to set off the tranquility, freshness and cleanness, and girls look back with a smile on a stone arch bridge, shading the light emptiness and mood[8]. Works of profound artistic conception also requires to permeate expressions of feeling and body moods, rationally combine spatial flow and action body, and contrast fast and slow rhythms, long and short rhythms, relaxed and urgent rhythms, and heavy and light rhythms. For example, for the Xie's Clog, dancers suddenly sigh while breathing smoothly, suddenly pause while turning around, grab while throwing off sleeves, walk mainly by lifting legs and shaking knees, and make action stroke on and off, to create an artistic conception of being cute, aloof, proud, natural, unrestrained, elegant, and lingering among mountains and rivers. Again, Han and Tang dances contain a lot of weightlessness and inclination like leaning tower rushing forward or upward, letting dancers or audiences enjoy magnificence and easiness. The dancing postures are full of youthful spirit, are vigorous, have magnificent, profound, simple and vigorous artistic conception, reflecting the vitality and entelechy in the Han and Tang dynasties and era ethos and spirit.

\section{Conclusion}

To sum up, Chinese classical dance is a perfect unity of rhythm, power, spirit and appearance. The dance body is to gasify and materialize body. This reflects clearing the mind and smelling scenes, drawing color adhere to the type, stream of life, and material pictographic culture. The movement path of body is sufficient to show dance language logic. The aesthetic implication should be concise and comprehensive, multiple styles, artistic conceptions, cultural connotations, types and schools 
should coexist. The dance style should show distinct character, feelings, manner, and model, to commit to spread the historical classics of Chinese people, advocate the core value of national spirit, and continue the everlasting national spirit. For creating Chinese classical dance in a personalized way, it should be ensured that the dance form contains classical beauty and artistic conception, and manifest the pursuant spiritual realm. It is feasible to extract materials from literature historical materials and historical stories, provided that cultural roots should be preserved, and sensitive artistic thought should be stimulated to make dance language with dynamic elements, expression elements and morphological elements. It is feasible to unify and interchange dance form and content, to present national fashion, morals, personalities and wisdom via dance works.

\section{References}

[1] Tian Tian, Theoretical Development History of Chinese Classical Dance (1954-2014)—Case Study of Dance, Journal of Beijing Dance Academy, Dance Symposium, Dance Art Series, and Dance Research, Ethnic Arts Studies, 2016(1):218-237.

[2] Zhou Minglong. Brief Analysis of Trend of "Neo-classicism" of Brigade Dance Creation, Dazhong Wenyi, 2017(1):167.

[3] Xu Wei. History Reflected in Jiangnan Dance Charm-Study on Character Image Shaping in the Ganjiang and Moxie, A Bing and Xi Shi, Hundred Schools in Arts, 2015(5):130-134.

[4] Wu Xiaoyun, Dance’s Wisdom Rests in Creation, Dance's Meaning Rests in Thought, Dance's Connotation Rests in Isomerism, and Dance's Implication and Charm Are Different-Discussion of Content and Form of Dance Creation, Modern Music, 2016(12):79-82.

[5] Yao Xiaoou, Wang Kejia. Analysis of the Gong Mo Dance and Early Drama Form Research Method in China-Interpretation of the Gong Mo Dance and Drama Form in the Han Dynasty, Journal of Yuxi Teachers' College, 2016,32(2):6-15.

[6] Shen Peiyi. Dawn Blossoms Plucked at Dusk-About Self-definition and Weighing of Dance Wors and Distance from Dancers to Drama Characters, Dance, 2016(6):34-37.

[7] Ma Fang. Discussion about the Tsinghua Bamboo Slips-Zhougong's Dance and "Confucias Cutting out Songs”-Discussion with Doc. Xie Bingjun, Academic Journal of Zhongzhou, 2016(7):145-150.

[8] Zhang Bo, Liu Junrong. Analysis of Mode and Idea of Integrating Nation Tradition and Culture with Physical Education-Case Study of the Dance of Picking Tea Leaves, Tea in Fujian, 2015(6):224-226. 